\title{
On the Security of a Bidirectional Proxy Re-encryption Scheme from PKC 2010
}

\author{
Jian Weng ${ }^{1,2,3}$, Yunlei Zhao ${ }^{4, \star}$, and Goichiro Hanaoka ${ }^{5}$ \\ 1 Department of Computer Science, Jinan University, Guangzhou, China \\ 2 State Key Laboratory of Networking and Switching Technology \\ Beijing University of Posts and Telecommunications, Beijing, China \\ 3 State Key Laboratory of Information Security \\ Institute of Software, Chinese Academy of Sciences, Beijing, China \\ 4 Software School, Fudan University, Shanghai, China \\ ${ }^{5}$ National Institute of Advanced Industrial Science and Technology, Tokyo, Japan \\ cryptjweng@gmail.com, yunleizhao@gmail.com, hanaoka-goichiro@aist.go.jp
}

\begin{abstract}
In ACM CCS 2007, Canetti and Hohenberger left an interesting open problem of how to construct a chosen-ciphertext secure proxy re-encryption (PRE) scheme without bilinear maps. This is a rather interesting problem and has attracted great interest in recent years. In PKC 2010, Matsuda, Nishimaki and Tanaka introduced a novel primitive named re-applicable lossy trapdoor function, and then used it to construct a PRE scheme without bilinear maps. Their scheme is claimed to be chosen-ciphertext secure in the standard model. In this paper, we make a careful observation on their PRE scheme, and indicate that their scheme does not satisfy chosen-ciphertext security. The purpose of this paper is to clarify the fact that, it is still an open problem to come up with a chosen-ciphertext secure PRE scheme without bilinear maps in the standard model.
\end{abstract}

Keywords: bilinear map, proxy re-encryption, chosen-ciphertext security, standard model.

\section{Introduction}

Proxy re-encryption (PRE), introduced by Blaze, Bleumer and Strauss [3] in Eurocrypt'98, allows a semi-trust proxy to translate a ciphertext intended for Alice into another ciphertext intended for Bob. The proxy, however, can not learn anything about the underlying messages. According to the direction of transformation, PRE can be categorized into bidirectional PRE, in which the proxy can transform ciphertexts from Alice to Bob and vice versa, and unidirectional $\mathrm{PRE}$, in which the proxy cannot transform ciphertexts in the opposite direction. PRE can also be categorized into multi-hop PRE, in which the ciphertexts can be transformed from Alice to Bob and then to Charlie and so on, and single-hop PRE, in which the ciphertexts can only be transformed once.

\footnotetext{
^ Corresponding author.
} 
In their seminal paper, Blaze et al. 3. proposed the first bidirectional PRE scheme. Ateniese et al. [1,2] presented unidirectional PRE schemes from bilinear maps. All of these schemes are only secure against chosen-plaintext attacks (CPA). However, applications often require security against chosen-ciphertext attacks (CCA).

To fill this gap, Canetti and Hohenberger 7] presented the first CCA-secure bidirectional multi-hop PRE scheme in the standard model. Libert and Vergnaud [14,13 proposed a unidirectional single-hop PRE scheme, which is replayable CCA-secure [8] in the standard model. Recently, Weng et al. 18] presented a unidirectional single-hop PRE scheme, which is CCA-secure against adaptive corruption of users in the standard model. These schemes rely on bilinear maps. In spite of the recent advances in implementation technique, compared with standard operations such as modular exponentiation in finite fields, the bilinear map computation is still considered as a rather expensive operation. It would be desirable for cryptosystems to be constructed without relying on pairings, especially in computation resource limited settings. Thus, in ACM CCS'07, Canetti and Hohenberger [7] left an open problem of how to construct a CCA-secure PRE scheme without bilinear maps.

Deng et al. 10, 19 presented a bidirectional single-hop PRE scheme without bilinear maps, and proved its CCA-security in the random oracle model. Shao et al. 17] presented a unidirectional single-hop PRE scheme without bilinear maps in the random oracle model, but their scheme was later identified a security flaw in [9]. Sherman et al. 9] presented a CCA-secure unidirectional single-hop PRE scheme without bilinear maps, again in the random oracle model. It is well-known [5, 6] that a proof in the random oracle model can only serve as an argument, which does not imply the security for real implementations. Thus, it is more desirable to come up with a CCA-secure PRE scheme without bilinear maps in the standard model.

In PKC 2010, Matsuda, Nishimaki and Tanaka made an important step and tried to construct such a scheme. They first introduced a new cryptographic primitive named re-applicable lossy trapdoor functions (re-applicable LTDFs), which are specialized lossy trapdoor functions 16,11,4, 12, and then used this primitive to construct a PRE scheme without bilinear maps. They claimed that their scheme is CCA-secure in the standard model. However, in this paper, we present a concrete attack, and indicate that their PRE scheme does not achieve the CCA-security. However, we stress that Matsuda et al.'s work is still considered as an important step in this research area. Namely, due to their scheme, we can figure out one of main difficulties for constructing CCA-secure PRE without using bilinear maps, and this would enable us to further design novel schemes which overcome the same problem.

\section{Preliminaries}

The Matsuda-Nishimaki-Tanaka PRE scheme involves the primitives of all-butone trapdoor function and re-applicable $(n, k)$ lossy trapdoor functions (LTDFs). 
Thus in this section, we shall review the definitions of these two primitives (for more details, the reader is referred to [15] and [16]). We shall also review the definition and security notion for bidirectional multi-hop PRE.

\subsection{All-but-One Trapdoor Function}

Let $B=\left\{B_{\lambda}\right\}_{\lambda \in \mathbb{N}}$ be a collection of sets whose elements represents the branches. A collection of $(n, k)$-all-but-one trapdoor functions is a tuple of probabilistic polynomial time $(\mathrm{PPT})$ algorithms $\left(\mathrm{G}_{\mathrm{abo}}, \mathrm{F}_{\mathrm{abo}}, \mathrm{F}_{\mathrm{abo}}^{-1}\right)$ having the following properties:

- All-but-one property: Given a lossy branch $b^{*} \in B_{\lambda}$, algorithm $\mathrm{G}_{\mathrm{abo}}\left(1^{\lambda}, b^{*}\right)$ outputs a pair $(s, t d)$, where $s$ is a function index and $t d$ is its trapdoor. For any $b \in B_{\lambda} \backslash\left\{b^{*}\right\}$, the algorithm $\mathrm{F}_{\text {abo }}(s, b, \cdot)$ computes an injective function $f_{s, b}(\cdot)$ over $\{0,1\}^{n}$, and $\mathrm{F}_{\text {abo }}^{-1}(t d, b, \cdot)$ computes $f_{s, b}^{-1}(\cdot)$. For the lossy branch $b^{*}, \mathrm{~F}_{\text {abo }}\left(s, b^{*}, \cdot\right)$ computes a lossy function $f_{s, b^{*}}(\cdot)$ over the domain $\{0,1\}^{n}$, where $\left|f_{s, b^{*}}\left(\{0,1\}^{n}\right)\right| \leq 2^{n-k}$.

- Indistinguishability: For every $b_{1}^{*}$ and $b_{2}^{*} \in B_{\lambda}$, the first output $s_{0}$ of $\mathrm{G}_{\mathrm{abo}}\left(1^{\lambda}, b_{0}^{*}\right)$ and the first output $s_{1}$ of $\mathrm{G}_{\mathrm{abo}}\left(1^{\lambda}, b_{1}^{*}\right)$ are computationally indistinguishable.

\subsection{Re-applicable $(n, k)$-Lossy Trapdoor Functions}

A collection of re-applicable $(n, k)$-lossy trapdoor functions (LTDFs) with respect to function indices is a tuple of PPT algorithms (ParGen, LossyGen, LossyEval, Lossylnv, Relndex, ReEval, PrivReEval, Trans, FakeKey) such that:

Injectivity: For every public parameter par $\leftarrow \operatorname{ParGen}\left(1^{\lambda}\right)$ and every $\operatorname{tag} \tau \in$ $\mathcal{T} \backslash\left\{\tau_{\text {los }}\right\}$, LossyGen $(\tau)$ outputs a pair of a function index and its trapdoor $(s, t d)$, LossyEval $(s, \cdot)$ computes an injective function $f_{s, \tau}(\cdot)$ over $\{0,1\}^{n}$, and Lossylnv $(t d, \tau, \cdot)$ computes $f_{s, \tau}^{-1}(\cdot)$. (We represent the function $f_{s, \tau}$, not $f_{s}$, in order to clarify a tag $\tau$. If we do not need to clarify a tag, we represent a function as $\left.f_{s, \star}\right)$.

Lossiness: For every public parameter par $\leftarrow \operatorname{ParGen}\left(1^{\lambda}\right)$, LossyGen $\left(\tau_{\text {los }}\right)$ outputs $(s, \perp)$ and LossyEval $(s, \cdot)$ computes a function $f_{s, \tau_{\text {los }}}(\cdot)$ over $\{0,1\}^{n}$, where $\left|f_{s, \tau_{\text {los }}}\left(\{0,1\}^{n}\right)\right| \leq 2^{n-k}$.

Indistinguishability between injective and lossy indices: Let $X_{\lambda}$ denote the distribution of (par, $\left.s_{\mathrm{inj}}, \tau\right)$, and $Y_{\lambda}$ denote the distribution of ( $\left.p a r, s_{\mathrm{los}}, \tau^{\prime}\right)$, where par is a public parameter from $\operatorname{ParGen}\left(1^{\lambda}\right), \tau$ and $\tau^{\prime}$ are random elements in $\mathcal{T}$, and the function indices $s_{\text {inj }}$ and $s_{\text {los }}$ are the first element output from LossyGen $(\tau)$ and LossyGen $\left(\tau_{\text {los }}\right)$ respectively. Then, $\left\{X_{\lambda}\right\}$ and $\left\{Y_{\lambda}\right\}$ are computationally indistinguishable.

Re-applying with respect to function indices: Let $\tau_{i}$ and $\tau_{j}$ be any tags with $\tau_{i} \neq \tau_{\text {los }}$ and $\tau_{j} \neq \tau_{\text {los }}$. The algorithm Relndex $\left(t d_{i}, t d_{j}\right)$ outputs $s_{i \leftrightarrow j}$, where $t d_{i}$ and $t d_{j}$ are the second elements of LossyGen $\left(\tau_{i}\right)$ and LossyGen $\left(\tau_{j}\right)$. Then, for any $x \in\{0,1\}^{n}, x=\operatorname{Lossylnv}\left(t d_{j}, \tau_{i}, \operatorname{ReEval}\left(s_{i \leftrightarrow j}, \operatorname{LossyEval}\left(s_{i}, x\right)\right)\right)$. Note that Lossylnv takes $\tau_{i}$ as one of the inputs, not $\tau_{j}$. 
Generating proper outputs: Let $c$ be an output from $\operatorname{ReEval}\left(s_{i \leftrightarrow j}\right.$, LossyEval $\left.\left(s_{i}, x\right)\right)$, where $s_{i \leftrightarrow j}$ and $s_{i}$ have the same meaning as that in the above paragraph. Then, PrivReEval $\left(x, \tau_{i}, \tau_{j}, s_{j}\right)$ outputs the same $c$, where $x$, $\tau_{i}, \tau_{j}$, and $s_{j}$ have the same meaning as that in the above paragraph. That is, ReEval $\left(s_{i \leftrightarrow j}, \operatorname{LossyEval}\left(s_{i}, \cdot\right)\right)$ and PrivReEval $\left(\cdot, \tau_{i}, \tau_{j}, s_{j}\right)$ are equivalent as a function (i.e. any output of $\operatorname{ReEval}\left(s_{i \leftrightarrow j}, \operatorname{LossyEval}\left(s_{i}, \cdot\right)\right)$ is independent of $s_{i}$ ).

Transitivity: Let $\left(s_{i}, t d_{i}\right),\left(s_{j}, t d_{j}\right)$ and $\left(s_{k}, t d_{k}\right)$ be outputs from LossyGen $\left(\tau_{i}\right)$, LossyGen $\left(\tau_{j}\right)$, and LossyGen $\left(\tau_{k}\right)$, and let $s_{i \leftrightarrow j}$ and $s_{i \leftrightarrow k}$ be the outputs from Relndex $\left(t d_{i}, t d_{j}\right)$ and Relndex $\left(t d_{i}, t d_{k}\right)$, respectively. Then, Trans $\left(s_{i \leftrightarrow j}, s_{i \leftrightarrow k}\right)$ outputs $s_{j \leftrightarrow k}$ which is the same output from Relndex $\left(t d_{j}, t d_{k}\right)$.

Fake key statistical indistinguishability: The algorithm FakeKey $\left(s_{i}, \tau_{i}\right)$ outputs $\left(s_{j}^{\prime}, s_{i \leftrightarrow j}^{\prime}, \tau_{j}^{\prime}\right)$, where $s_{i}$ is the first element of an output from $\operatorname{LossyGen}\left(\tau_{i}\right)$. Let $X_{\lambda}$ denote the distribution of ( $\operatorname{par}, s_{i}, s_{j}, s_{i \leftrightarrow j}, \tau_{i}, \tau_{j}$ ), and let $Y_{\lambda}$ denote the distribution of ( $\mathrm{par}, s_{i}, s_{j}^{\prime}, s_{i \leftrightarrow j}^{\prime}, \tau_{i}, \tau_{j}^{\prime}$ ), where each par, $s_{j}, s_{i \leftrightarrow j}$, and $\tau_{j}$ has the same meaning as that in the above paragraph. Then, $\left\{X_{\lambda}\right\}$ and $\left\{Y_{\lambda}\right\}$ are statistically indistinguishable.

Generation of injective functions from lossy functions: Let $s$ be the first element of an output from FakeKey $\left(s_{\operatorname{los}}, \tau\right)$, where $\tau$ is a tag and $s_{\text {los }}$ is the first element of an output from LossyGen $\left(\tau_{\text {los }}\right)$. Then, for every $\tau, \operatorname{LossyEval}(s, \cdot)$ represents an injective function $f_{s, \star}$ with overwhelming probability, where a random variable is the randomness of FakeKey $\left(s_{\text {los }}, \tau\right)$. (We do not require other properties of index $s$ if $f_{s, \star}$ is injective. The function $f_{s, \star}$ cannot have any trapdoor information).

\subsection{Realization of Re-applicable LTDFs}

Based on Peikert and Waters' LTDFs [16], Matsuda, Nishimaki and Tanaka [15] gave a realization of re-applicable LTDFs, which is specified as below (for more details, the reader is referred to [15]):

ParGen: This algorithm first generates a cyclic group $\mathbb{G}$ with prime order $p$, and then chooses a random generator $g \in \mathbb{G}$. Next, it selects random numbers $r_{1}, \cdots, r_{n} \in R \mathbb{Z}_{p}$, and outputs the public parameters $\mathbf{C}_{1}$ as

$$
\mathbf{C}_{1}=\left(\begin{array}{c}
c_{1} \\
\vdots \\
c_{n}
\end{array}\right)=\left(\begin{array}{c}
g^{r_{1}} \\
\vdots \\
g^{r_{n}}
\end{array}\right)
$$

LossyGen: Taking as input the public parameter $\mathbf{C}_{1}$ and a tag $\tau \in \mathbb{G}$ (note that if $\tau$ is the identity element $e$ of $\mathbb{G}$, it means execution of the lossy mode; otherwise, execution of the injective mode), this algorithm first selects random elements $z_{1}, z_{2}, \cdots, z_{n} \in_{R} \mathbb{Z}_{p}$, and then computes a function index as 
$\mathbf{C}_{2}=\left(\begin{array}{ccc}c_{1,1} & \cdots & c_{1, n} \\ \vdots & \ddots & \vdots \\ c_{n, 1} & \cdots & c_{n, n}\end{array}\right)=\left(\begin{array}{ccc}c_{1}^{z_{1}} \cdot \tau & \cdots & c_{1}^{z_{n}} \\ \vdots & \ddots & \vdots \\ c_{n}^{z_{1}} & \cdots & c_{n}^{z_{n}} \cdot \tau\end{array}\right)= \begin{cases}c_{i, j}=c_{i}^{z_{j}} \cdot \tau, & \text { if } i=j \\ c_{i, j}=c_{i}^{z_{j}}, & \text { otherwise }\end{cases}$

Finally, it outputs the function index $s=\left(\mathbf{C}_{1}, \mathbf{C}_{2}\right)$ and the trapdoor $t d=$ $z=\left(z_{1}, \cdots, z_{n}\right)$.

LossyEval: Taking as input a function index $s=\left(C_{1}, C_{2}\right)$ and an $n$-bit input $\mathbf{x}=\left(x_{1}, \cdots, x_{n}\right) \in\{0,1\}^{n}$, this algorithm outputs $\left(y_{1}, \mathbf{y}_{2}\right)$ such that

$$
\begin{aligned}
& y_{1}=\mathbf{x C}_{1}=\prod_{i=1}^{n} c_{i}^{x_{i}}, \\
& \mathbf{y}_{2}=\mathbf{x C}_{2}=\left(\prod_{i=1}^{n} c_{i, 1}^{x_{i}}, \cdots, \prod_{i=1}^{n} c_{i, n}^{x_{i}}\right)=\left(\left(\prod_{i=1}^{n} c_{i}^{z_{1} x_{i}}\right) \tau^{x_{1}}, \cdots,\left(\prod_{i=1}^{n} c_{i}^{z_{n} x_{i}}\right) \tau^{x_{n}}\right) .
\end{aligned}
$$

Lossylnv: Taking as input $\left(t d, \tau,\left(y_{1}, \mathbf{y}_{2}\right)\right)$, where the trapdoor information $t d$ consists of $z=\left(z_{1}, \cdots, z_{n}\right)$, the tag $\tau$ is an element in $\mathbb{G} \backslash\{e\}$, and $\mathbf{y}_{2}=$ $\left(y_{2,1}, \cdots, y_{2, n}\right) \in \mathbb{G}^{1 \times n}$, this algorithm computes $\mathbf{w}=\left(y_{2,1} \cdot y_{1}^{-z_{1}}, y_{2,2}\right.$. $\left.y_{1}^{-z_{2}}, \cdots, y_{2, n} \cdot y_{1}^{-z_{n}}\right)$. Then, if $j$-th element of $\mathbf{w}$ is the identity element of $\mathbb{G}$, then it sets $x_{j}=0$; else if the $j$-th element of $\mathbf{w}$ is $\tau$ then it sets $x_{j}=1$; otherwise, it outputs $\perp$. Finally, it outputs $x=\left(x_{1}, \cdots, x_{n}\right)$.

Relndex: Taking as input trapdoors $t d_{i}=\left(z_{1}, \cdots, z_{n}\right)$ and $t d_{j}=\left(z_{1}^{\prime}, \cdots, z_{n}^{\prime}\right)$, this algorithm outputs $s_{i \leftrightarrow j}=t d_{j}-t d_{i}=\left(z_{1}^{\prime}-z_{1}, \cdots, z_{n}^{\prime}-z_{n}\right)=\left(z_{1, i \leftrightarrow j}, \cdots\right.$, $\left.z_{n, i \leftrightarrow j}\right)$.

ReEval: On input $\left(s_{i \leftrightarrow j},\left(y_{1}, \mathbf{y}_{2}\right)\right)$, where $s_{i \leftrightarrow j}=\left(z_{1, i \leftrightarrow j}, z_{2, i \leftrightarrow j}, \cdots, z_{n, i \leftrightarrow j}\right)$ and $\left(y_{1}, \mathbf{y}_{2}\right)=\left(y_{1},\left(y_{2,1}, y_{2,2}, \cdots, y_{2, n}\right)\right)$, this algorithm computes $\mathbf{y}_{2}^{\prime}=\left(y_{2,1}^{\prime}, y_{2,2}^{\prime}\right.$, $\left.\cdots, y_{2, n}^{\prime}\right)=\left(y_{2,1} \cdot y_{1}^{z_{1, i \leftrightarrow j}}, y_{2,2} \cdot y_{1}^{z_{2, i \leftrightarrow j}}, \cdots, y_{2, n} \cdot y_{1}^{z_{n, i \leftrightarrow j}}\right)$. Then it outputs $\left(y_{1}, \mathbf{y}_{2}^{\prime}\right)$.

PrivReEval: Taking as input $\mathbf{x}, \tau_{i}, \tau_{j}$ and $s_{j}$, where $\mathbf{x}=\left(x_{1}, \cdots, x_{n}\right)$ is $n$-bit input, this algorithm first computes $\left(\hat{y}_{1}, \hat{\mathbf{y}}_{2}\right) \leftarrow \operatorname{LossyEval}\left(s_{j}, \mathbf{x}\right)$. Next, it makes $\hat{\mathbf{y}}_{2}^{\prime}$ from $\hat{\mathbf{y}}_{2}$ in the following process: for each $i \in[1, n]$, if $x_{i}=1$ then $\hat{y}_{2, i}^{\prime}=\hat{y}_{2, i} \tau_{j}^{-1} \tau_{i}$; else $\hat{y}_{2, i}^{\prime}=\hat{y}_{2, i}$, where $\hat{y}_{2, i}$ and $\hat{y}_{2, i}^{\prime}$ are the $i$-th elements of $\hat{\mathbf{y}}_{2}$ and $\hat{\mathbf{y}}_{2}^{\prime}$ respectively. Finally, it outputs $\left(\hat{y}_{1}, \hat{\mathbf{y}}_{2}^{\prime}\right)$.

Trans: Taking as input $s_{i \leftrightarrow j}$ and $s_{i \leftrightarrow k}$, this algorithm outputs $s_{i \leftrightarrow k}-s_{i \leftrightarrow j}=$ $\left(t d_{k}-t d_{i}\right)-\left(t d_{j}-t d_{i}\right)=t d_{k}-t d_{j}=s_{i \leftrightarrow k}$.

FakeKey: Taking as input a function index $s_{i}=\left(\mathbf{C}_{1}, \mathbf{C}_{2}\right)$ and a $\operatorname{tag} \tau_{i} \in \mathbb{G}$, this algorithm first chooses a random element $t \in \mathbb{G}$. Next, it chooses random numbers $s_{i \leftrightarrow j}=\left(z_{1, i \leftrightarrow j}, \cdots, z_{n, i \leftrightarrow j}\right) \in_{R} \mathbb{Z}_{p}^{n}$. Then it makes a new matrix $\mathbf{C}_{2}^{\prime}$ as follows:

$$
\mathbf{C}_{2}^{\prime}=\left(\begin{array}{ccc}
c_{1,1} \cdot c_{1}^{z_{1, i \leftrightarrow j}} \cdot t \cdots & c_{1, n} \cdot c_{1}^{z_{n, i \leftrightarrow j}} \\
\vdots & \ddots & \vdots \\
c_{n, 1} \cdot c_{n}^{z_{1, i \leftrightarrow j}} & \cdots & c_{n, n} \cdot c_{n}^{z_{n, i \leftrightarrow j}} \cdot t
\end{array}\right)=\left\{\begin{array}{c}
c_{k, \ell}^{\prime}=c_{k, \ell} \cdot c_{k}^{z_{\ell, i \leftrightarrow j}} \cdot t, \text { if } k=\ell ; \\
c_{k, \ell}^{\prime}=c_{k, \ell} \cdot c_{k}^{z_{\ell, i \leftrightarrow j}}, \quad \text { otherwise, }
\end{array}\right.
$$

where $c_{k}$ is the $k$ entry of $\mathbf{C}_{1}$, and $c_{k, \ell}$ is the $(k, \ell)$ entry of $\mathbf{C}_{2}$. Finally, it outputs $s_{j}=\left(\mathbf{C}_{1}, \mathbf{C}_{2}^{\prime}\right), s_{i \leftrightarrow j}=\left(z_{1, i \leftrightarrow j}, \cdots, z_{n, i \leftrightarrow j}\right)$ and $\tau_{j}=\tau_{i} \cdot t$. 


\section{Bidirectional Multi-hop PRE}

A bidirectional PRE scheme $\Pi=$ (Setup, KeyGen, Enc, ReKeyGen, ReEnc, Dec) consists of the following six algorithms:

- Setup $\left(1^{\lambda}\right)$ : Given a security parameter $1^{\lambda}$, this setup algorithm outputs a public parameter $P P$. Denote this by $P P \leftarrow \operatorname{Setup}\left(1^{\lambda}\right)$.

- KeyGen $(P P)$ : Given the public parameter $P P$, this key generation algorithm outputs a public key $p k$ and a secret key $s k$. Denote this by $(p k, s k) \leftarrow$ $\operatorname{KeyGen}(P P)$.

- $\operatorname{Enc}(P P, p k, m)$ : Given the public parameter $P P$, a public key $p k$ and a message $m$ in the message space $\mathcal{M}$, this encryption algorithm outputs a ciphertext $C$. Denote this by $C \leftarrow \operatorname{Enc}(P P, p k, m)$.

- ReKeyGen $\left(P P, s k_{i}, s k_{j}\right)$ : Given the public parameter $P P$, a pair of secret keys $s k_{i}$ and $s k_{j}$ where $i \neq j$, this re-encryption key generation algorithm outputs a re-encryption key $r k_{i \leftrightarrow j}$. Denote this by $r k_{i \leftrightarrow j} \leftarrow \operatorname{ReKeyGen}\left(P P, s k_{i}, s k_{j}\right)$.

- $\operatorname{Re} \operatorname{Enc}\left(P P, r k_{i \leftrightarrow j}, C_{i}\right)$ : Given the public parameter $P P$, a re-encryption key $r k_{i \leftrightarrow j}$ and a ciphertext $C_{i}$ intended for user $i$, this re-encryption algorithm outputs another ciphertext $C_{j}$ for user $j$ or the error symbol $\perp$. Denote this by $C_{j} \leftarrow \operatorname{Re} \operatorname{Enc}\left(P P, r k_{i \leftrightarrow j}, C_{i}\right)$.

- $\operatorname{Dec}(P P, s k, C)$ : Given the public parameter $P P$, a public key $s k$ and a ciphertext $C$, this decryption algorithm outputs a message $m$ or the error symbol $\perp$.

Next, we review the definition of chosen-ciphertext security for bidirectional multi-hop PRE scheme as defined in [15, 7]. Let $\lambda$ be the security parameter, $\mathcal{A}$ be an oracle TM, representing the adversary, and $\Gamma_{U}$ and $\Gamma_{C}$ be two lists which are initially empty. The game consists of an execution of $\mathcal{A}$ with the following oracles, which can be invoked multiple times in any order, subject to the constraints specified as below:

Setup Oracle: This oracle can be queried first in the game only once. This oracle generates the public parameters $P P \leftarrow \operatorname{Setup}\left(1^{\lambda}\right)$, and gives $P P$ to $\mathcal{A}$.

Uncorrupted key generation: This oracle first generates a new key pair by running $(p k, s k) \leftarrow \operatorname{KeyGen}(P P)$. Next, it adds $p k$ in $\Gamma_{U}$, and gives $p k$ to $\mathcal{A}$.

Corrupted key generation: This oracle generates a new key pair by running $(p k, s k) \leftarrow$ KeyGen $(P P)$. Next, it adds $p k$ in $\Gamma_{C}$, and gives $(p k, s k)$ to $\mathcal{A}$.

Challenge oracle: This oracle can be queried only once. On input $\left(p k_{i^{*}}, m_{0}\right.$, $\left.m_{1}\right)$, this oracle randomly chooses a bit $b \in\{0,1\}$ and gives $C_{i^{*}}=\operatorname{Enc}(P P$, $\left.p k_{i^{*}}, m_{b}\right)$ to $\mathcal{A}$. Here it is required that $p k_{i^{*}} \in \Gamma_{U}$. We call $p k_{i^{*}}$ the challenge key and $C_{i^{*}}$ the challenge ciphertext.

Re-encryption key generation: On input $\left(p k_{i}, p k_{j}\right)$ from the adversary, this oracle gives the re-encryption key $r k_{i \leftrightarrow j}=\operatorname{ReKeyGen}\left(P P, s k_{i}, s k_{j}\right)$ to $\mathcal{A}$, where $s k_{i}$ and $s k_{j}$ are the secret keys corresponding to $p k_{i}$ and $p k_{j}$, respectively. Here it is required that $p k_{i}$ and $p k_{j}$ are both in $\Gamma_{C}$, or alternatively are both in $\Gamma_{U}$. 
Re-encryption oracle: On input $\left(p k_{i}, p k_{j}, C_{i}\right)$, if $p k_{j} \in \Gamma_{C}$ and $\left(p k_{i}, C_{i}\right)$ is a derivative of $\left(p k_{i^{*}}, C_{i^{*}}\right)$, this oracle give $\mathcal{A}$ a special symbol $\perp$, which is not in the domain of messages or ciphertext. Otherwise, it gives the re-encrypted ciphertext $C_{j}=\operatorname{ReEnc}\left(P P, \operatorname{ReKeyGen}\left(P P, s k_{i}, s k_{j}\right), C_{i}\right)$ to $\mathcal{A}$. Derivatives of $\left(p k_{i^{*}}, C_{i^{*}}\right)$ are defined inductively as follows:

$-\left(p k_{i^{*}}, C_{i^{*}}\right)$ is a derivative of itself.

- If $(p k, C)$ is a derivative of $\left(p k_{i^{*}}, C_{i^{*}}\right)$, and $\left(p k^{\prime}, C^{\prime}\right)$ is a derivative of $(p k, C)$, then $\left(p k^{\prime}, C^{\prime}\right)$ is a derivative of $\left(p k_{i^{*}}, C_{i^{*}}\right)$.

- If $\mathcal{A}$ has queried the re-encryption oracle on input $\left(p k, p k^{\prime}, C\right)$ and obtained the response $C^{\prime}$, then $\left(p k^{\prime}, C^{\prime}\right)$ is a derivative of $(p k, C)$.

- If $\mathcal{A}$ has queried the re-encryption key generation oracle on input $\left(p k, p k^{\prime}\right)$ or $\left(p k^{\prime}, p k\right)$, and $C^{\prime}=\operatorname{ReEnc}\left(P P, \operatorname{ReKeyGen}\left(P P, s k, s k^{\prime}\right), C\right)$, then $\left(p k^{\prime}, C^{\prime}\right)$ is a derivative of $(p k, C)$, where $s k$ and $s k^{\prime}$ are the secret keys corresponding to $p k$ and $p k^{\prime}$, respectively.

Decryption oracle: On input $(p k, C)$, if the pair $(p k, C)$ is a derivative of the challenge key and ciphertext $\left(p k_{i^{*}}, C_{i^{*}}\right)$, or $p k$ is not in $\Gamma_{U} \cup \Gamma_{C}$, this oracle returns the special symbol $\perp$ to $\mathcal{A}$. Otherwise, it returns the result of $\operatorname{Dec}(P P, s k, C)$ to $\mathcal{A}$, where $s k$ is the secret key with respect to $p k$.

Decision oracle: This oracle can be queried at the end of the game. On input $b^{\prime}$, if $b^{\prime}=b$ and the challenge key $p k_{i^{*}} \in \Gamma_{U}$, this algorithm output 1 ; else output 0 .

We describe the output of the decision oracle in the above CCA-security definitional game as $\operatorname{Expt}_{\Pi, \mathcal{A}}^{\text {bid-PRE-CCA }}(\lambda)=b$ for an adversary $A$ and a scheme $\Pi$. We define the advantage of adversary $\mathcal{A}$ as

$$
\operatorname{Adv}_{\Pi, \mathcal{A}}^{\text {bid-PRE-CCA }}(\lambda) \stackrel{\text { def }}{=}\left|\operatorname{Pr}\left[\operatorname{Expt}_{\Pi, \mathcal{A}}^{\text {bid-PRE-CCA }}(\lambda)=1\right]-\frac{1}{2}\right|,
$$

where the probability is over the random choices of $\mathcal{A}$ and oracles. We say that the scheme $\Pi$ is secure under the bidirectional PRE-CCA attack, if for any PPT adversary $A$, his advantage $\operatorname{Adv}_{\Pi, \mathcal{A}}^{\text {bid-PRE-CCA }}(\lambda)$ is negligible in the security parameter $\lambda$ (for sufficiently large $\lambda$ ).

\section{Review of the Matsuda-Nishimaki-Tanaka PRE Scheme}

In this section, we shall review the Matsuda-Nishimaki-Tanaka bidirectional multi-hop PRE scheme.

Let $\lambda$ be the security parameter, and let $n, k, k^{\prime}, k^{\prime \prime}$ and $v$ be parameters depended on $\lambda$. Let (SigGen, SigSign, SigVer) be a strongly unforgeable one-time signature scheme where the verification keys are in $\{0,1\}^{v}$. Let (ParGen, LossyGen, LossyEval, Lossylnv, ReEval, PrivReEval, Trans, FakeKey) be a collection of reapplicable $(n, k)$-LTDFs and $\mathcal{T}$ be a set of tags. Let $\left(\mathrm{G}_{\text {abo }}, \mathrm{F}_{\text {abo }}, \mathrm{F}_{\text {abo }}^{-1}\right)$ be a collection of $\left(n, k^{\prime}\right)$-ABO trapdoor functions with branches $B_{\lambda}=\{0,1\}^{v}$, which contains the set of signature verification keys. Let $\mathcal{H}$ be a family of pairwise independent hash functions from $\{0,1\}^{n}$ to $\{0,1\}^{k^{\prime \prime}}$. It is required that the above parameters 
satisfy $\left(k+k^{\prime}\right)-\left(k^{\prime \prime}+n\right) \geq \delta=\delta_{1}+\delta_{2}$ for some $\delta_{1}=\omega(\log \lambda)$ and $\delta_{2}=\omega(\log \lambda)$. The message space of the system is $\{0,1\}^{k^{\prime \prime}}$. The Matsuda-Nishimaki-Tanaka PRE scheme [15] is specified by the following algorithms:

Setup $\left(1^{\lambda}\right)$ : This algorithm first generates an index of all-but-one trapdoor functions with lossy branch $0^{v}:\left(s_{\mathrm{abo}}, t d_{\mathrm{abo}}\right) \leftarrow \mathrm{G}_{\mathrm{abo}}\left(1^{\lambda}, 0^{v}\right)$. Then, it generates a public parameter of re-applicable LTDFs: par $\leftarrow \operatorname{ParGen}\left(1^{\lambda}\right)$. Next, it chooses a hash function $h$ from $\mathcal{H}$. Finally, it outputs a public parameter as $P P=\left(s_{\text {abo }}\right.$, par, $\left.h\right)$.

Note that the algorithm Setup erases the trapdoor $t d_{\text {abo }}$ because the following algorithms do not use $t d_{\text {abo }}$.

KeyGen $(P P)$ : Taking as input the pubic parameters $P P=\left(s_{\text {abo }}\right.$, par, $\left.h\right)$, this algorithm first chooses a tag $\tau \in \mathcal{T} \backslash\left\{\tau_{\text {los }}\right\}$ and generates an injective index of re-applicable LTDFs: $\left(s_{\text {rltdf }}, t d_{\text {rltdf }}\right) \leftarrow$ LossyGen $(\tau)$. Finally, it outputs the public key $p k=\left(s_{\text {rltdf }}, \tau\right)$ and the secret key $s k=\left(t d_{\text {rltdf }}, s_{\text {rltdf }}, \tau\right)$.

Enc $(P P, p k, m)$ : Taking as input the public parameters $P P=\left(s_{\text {abo }}\right.$, par, $\left.h\right)$, public key $p k=\left(s_{\text {rltdf }}, \tau\right)$ and a message $m \in\{0,1\}^{k^{\prime \prime}}$, this encryption algorithm first chooses $x \in\{0,1\}^{n}$ uniformly at random. Next it generates a key-pair for the one-time signature scheme: $\left(v k, s k_{\sigma}\right) \leftarrow \operatorname{Sig} \operatorname{Gen}\left(1^{\lambda}\right)$, and computes

$$
c_{1}=\operatorname{LossyEval}\left(s_{\text {rltdf }}, x\right), c_{2}=\mathrm{F}_{\text {abo }}\left(s_{\text {abo }}, v k, x\right), c_{3}=h(x) \oplus m .
$$

Then it signs a tuple $\left(c_{2}, c_{3}, \tau\right)$ as $\sigma \leftarrow \operatorname{SigSign}\left(s k_{\sigma},(c 2, c 3, \tau)\right)$. Finally, it outputs the ciphertext $C=\left(v k, c_{1}, c_{2}, c_{3}, \tau, \sigma\right)$.

$\operatorname{ReKeyGen}\left(P P, s k_{i}, s k_{j}\right)$ : On public parameter $P P=\left(s_{\text {abo }}\right.$, par, $\left.h\right)$, the secret keys $s k_{i}=\left(t d_{i}, s_{i}, \tau_{i}\right)$ and $s k_{j}=\left(t d_{j}, s_{j}, \tau_{j}\right)$, this algorithm computes $s_{i \leftrightarrow j} \leftarrow$ Relndex $\left(t d_{i}, t d_{j}\right)$, and then outputs a re-encryption key $r k_{i \leftrightarrow j}=s_{i \leftrightarrow j}$.

$\operatorname{ReEnc}\left(P P, r k_{i \leftrightarrow j}, C_{i}\right)$ : Taking as input the public parameter $P P=\left(s_{\text {abo }}\right.$, par, $\left.h\right)$, the re-encryption key $r k_{i \leftrightarrow j}=s_{i \leftrightarrow j}$ and a ciphertext $C_{i}=\left(v k, c_{1, i}, c_{2}, c_{3}, \tau, \sigma\right)$, this algorithm computes $c_{1, j} \leftarrow \operatorname{ReEval}\left(s_{i \leftrightarrow j}, c_{1, i}\right)$. It then outputs $C_{j}=$ $\left(v k, c_{1, j}, c_{2}, c_{3}, \tau, \sigma\right)$ as a new ciphertext for the user with $s k_{j}$.

$\operatorname{Dec}(P P, s k, C)$ : Taking as input the public parameter $P P=\left(s_{\text {abo }}\right.$, par, $\left.h\right)$, a secret key $s k=\left(t d_{\text {rltdf }}, s_{\text {rltdf }}, \tau\right)$ and a ciphertext $C=\left(v k, c_{1}, c_{2}, c_{3}, \tau^{\prime}, \sigma\right)$, this decryption algorithm acts as follows:

1. Check whether $\operatorname{Sig} \operatorname{Ver}\left(v k,\left(c_{2}, c_{3}, \tau^{\prime}\right), \sigma\right)=1$ holds. If not, output $\perp$.

2. Compute $x=\operatorname{Lossylnv}\left(t d_{\text {rltdf }}, \tau^{\prime}, c_{1}\right)$. If $\tau=\tau^{\prime}$, it checks LossyEval $\left(s_{\text {rltdf }}, x\right)$ $=c_{1}$; else it checks PrivReEval $\left(x, \tau^{\prime}, \tau, s_{\text {rltdf }}\right)=c_{1}$. If not, it outputs $\perp$. It also checks $\mathrm{F}_{\text {abo }}\left(s_{\text {abo }}, v k, x\right)=c_{2}$. If not, it outputs $\perp$.

3. Finally, output $m=c_{3} \oplus h(x)$.

\section{$5 \quad$ Security Analysis}

In this section, we shall present a concrete attack against the Matsuda-NishimakiTanaka PRE scheme. Before presenting its details, we first identify the potential 
weakness in their scheme: for a ciphertext $C_{i}=\left(v k, c_{1, i}, c_{2}, c_{3}, \tau, \sigma\right)$, their ReEnc algorithm simply transforms the ciphertext component $c_{1, i}$ into $c_{1, j}$, without verifying the validity of $c_{1, i}$. Then there might exist an adversary who can break the CCA-security of their scheme as follows: Given the challenge ciphertext $C_{i^{*}}=$ $\left(v k, c_{1, i^{*}}, c_{2}, c_{3}, \tau, \sigma\right)$, the adversary can first modify the ciphertext component $c_{1, i^{*}}$ to obtain a new (ill-formed) ciphertext $C_{i^{*}}^{\prime}$ and then ask the re-encryption oracle to re-encrypt $C_{i^{*}}^{\prime}$ into another ciphertext $C_{j}^{\prime}$ for a corrupted user $j$ (note that according to the security model, it is legal for the adversary to issue such a query); next, the adversary can modify $C_{j}^{\prime}$ to obtain the right re-encrypted ciphertext $C_{j}$ of the challenge ciphertext, and thus he can derive the underlying plaintext by decrypting $C_{j}$ with user $j$ 's secret key.

Below we give the attack details. For an easy explanation of how the adversary can modify $C_{j}^{\prime}$ to obtain the right transformed ciphertext $C_{j}$, when describing the underlying re-applicable LTDFs we shall take Matsuda et al.'s concrete realization (recalled in Section 2.3) as the example. Concretely, the adversary works as follows:

1. The adversary first obtains the public parameters $P P$ from the setup oracle.

2 . The adversary obtains a public key $p k_{i^{*}}$ from the uncorrupted key generation oracle. Note that $p k_{i *}$ will be added in $\Gamma_{U}$ by the oracle.

3. The adversary obtains a public/secret key pair $\left(p k_{j}, s k_{j}\right)$ from the corrupted key generation oracle. Note that $p k_{j}$ will be added in $\Gamma_{C}$ by the oracle.

4. The adversary submits $\left(p k_{i^{*}}, m_{0}, m_{1}\right)$ to the challenge oracle, and then is given the challenge ciphertext $C_{i^{*}}=\left(v k^{*}, c_{1, i^{*}}, c_{2}^{*}, c_{3}^{*}, \tau^{*}, \sigma^{*}\right)$, where $c_{1, i^{*}}$ is the output of function LossyEval. Here we use Matsuda et al.'s concrete realization of LossyEval as an example. Wlog, suppose $c_{1, i^{*}}=\left(y_{1}, \mathbf{y}_{2}\right)=$ $\left(y_{1},\left(y_{2,1}, \cdots, y_{2, n}\right)\right)$.

5. The adversary first randomly picks $\tilde{y}_{2,1}, \cdots, \tilde{y}_{2, n}$ from $\mathbb{G}$, and modifies the challenge ciphertext to obtain a new (ill-formed) ciphertext $C_{i^{*}}^{\prime}=\left(v k^{*}, c_{1, i^{*}}^{\prime}\right.$, $\left.c_{2}^{*}, c_{3}^{*}, \tau^{*}, \sigma^{*}\right)$, where $c_{1, i^{*}}^{\prime}=\left(y_{1},\left(\tilde{y}_{2,1}, \cdots, \tilde{y}_{2, n}\right)\right)$. Then, the adversary submits $\left(p k_{i^{*}}, p k_{j}, C_{i^{*}}^{\prime}\right)$ to the re-encryption oracle. Note that, although $p k_{j} \in$ $\Gamma_{C}$, it is legal for the adversary to issue this query, since $\left(p k_{i^{*}}, C_{i^{*}}^{\prime}\right)$ is not a derivative of $\left(p k_{i^{*}}, C_{i^{*}}\right)$. Note that the re-encryption algorithm ReEnc cannot check the validity of the ciphertext component $c_{1, i^{*}}^{\prime}$. So, it will return the re-encrypted ciphertext $C_{j}^{\prime}=\operatorname{ReEnc}\left(P P, \operatorname{ReKeyGen}\left(P P, s k_{i^{*}}, s k_{j}\right), C_{i^{*}}^{\prime}\right)$ to the adversary.

According to the re-encryption algorithm, we get $C_{j}^{\prime}=\left(v k^{*}, c_{1, j}^{\prime}, c_{2}^{*}, c_{3}^{*}, \tau^{*}\right.$, $\left.\sigma^{*}\right)$, where $c_{1, j}^{\prime}=\operatorname{ReEval}\left(s_{i^{*} \leftrightarrow j}, c_{1, i^{*}}^{\prime}\right)$. According to Matsuda et al.'s concrete realization of ReEval, we have

$$
c_{1, j}^{\prime}=\left(y_{1},\left(\tilde{y}_{2,1}^{\prime}, \cdots, \tilde{y}_{2, n}^{\prime}\right)\right)=\left(y_{1},\left(\tilde{y}_{2,1} \cdot y_{1}^{z_{1, i * \leftrightarrow j}}, \cdots, \tilde{y}_{2, n} \cdot y_{1}^{z_{n, i * \leftrightarrow j}}\right)\right) .
$$

Now, from $c_{1, j}^{\prime}=\left(y_{1},\left(\tilde{y}_{2,1}^{\prime}, \cdots, \tilde{y}_{2, n}^{\prime}\right)\right)$, the adversary can compute the following 


$$
\begin{aligned}
c_{1, j} & =\left(y_{1},\left(\frac{\tilde{y}_{2,1}^{\prime} y_{2,1}}{\tilde{y}_{2,1}}, \cdots, \frac{\tilde{y}_{2, n}^{\prime} y_{2, n}}{\tilde{y}_{2, n}}\right)\right) \\
& =\left(y_{1},\left(\frac{\tilde{y}_{2,1} \cdot y_{1}^{z_{1, i^{*} \leftrightarrow j}} y_{2,1}}{\tilde{y}_{2,1}}, \cdots, \frac{\tilde{y}_{2, n} \cdot y_{1}^{z_{n, i^{*} \leftrightarrow j}} y_{2, n}}{\tilde{y}_{2, n}}\right)\right) \\
& =\left(y_{1},\left(y_{2,1} \cdot y_{1}^{z_{1, i^{*} \leftrightarrow j}}, \cdots, y_{2, n} \cdot y_{1}^{z_{n, i^{*} \leftrightarrow j}}\right)\right) .
\end{aligned}
$$

Observe that $c_{1, j}$ is indeed equivalent to the result of $\operatorname{ReEval}\left(s_{i^{*} \leftrightarrow j}, c_{1, i^{*}}\right)$. Thus, we have $C_{j}=\left(v k^{*}, c_{1, j}, c_{2}^{*}, c_{3}^{*}, \tau^{*}, \sigma^{*}\right)$ is indeed the result of $\operatorname{ReEnc}(P P$, $\left.\operatorname{ReKeyGen}\left(P P, s k_{i^{*}}, s k_{j}\right), C_{i^{*}}\right)$, which is an encryption of $m_{b}$. Now, the adversary can obtain the underlying plaintext $m_{b}$ by decrypting the re-encrypted ciphertext $C_{j}$ using the secret key $s k_{j}$, and obviously can break CCA-security of the Matsuda-Nishimaki-Tanaka PRE scheme.

The above attack can also be simply extended to the case that the user $j$ is uncorrupted. In this case, the adversary $\mathcal{A}$ directly request $\left(p k_{j}, C_{j}\right)$ to the decryption oracle, which will return the plaintext $m_{b}$ to $\mathcal{A}$.

\section{Discussions and Conclusion}

The authors constructed 11 games, Game-0 to Game-10, to prove the CCAsecurity of the PRE scheme developed in [15, where Game-0 is just the CCA definitional game of PRE (recalled in Section 3) and Game-10 is a game which any adversary can win with only probability $1 / 2$. They also discussed that difference of advantage between any two successive games is negligible, and hence any adversary cannot win Game- 0 with a better probability than $1 / 2$ plus a negligible value. However, as we observed in the previous section, there exists an adversary which can always win Game-0, and this implies that for at least one of pairs of two successive games, difference of advantage between them is nonnegligible. If we correctly understand the security proof in [15, such two games seem Game-7 and 8. This is basically due to the fact that until Game-7, the challenger generates all re-encryption keys for all users (including both uncorrupted and corrupted users), and by using these re-encryption keys, the challenger simulates both the re-encryption key generation oracle and the re-encryption oracle. In contrast, in Game-8, the challenger generates re-encryption keys among uncorrupted users in a specific manner without knowing these users' secret keys (see [15] for detail), and therefore, the same strategy for simulating these two oracles as in Game-7 cannot be immediately applied to this game. Namely, we see that in Game-8, it is still straightforward to generate re-encryption keys among uncorrupted users or those among corrupted users, but it seems hard to generate any re-encryption key between an uncorrupted user and a corrupted user. This also implies that the re-encryption key generation oracle can be still simulated, but the re-encryption oracle can not as long as the same simulation technique as in Game-7 is used. 
The PRE scheme developed in 15] is based upon the CCA-secure publickey encryption (PKE) scheme of Peikert and Waters (that is in turn based upon LTDFs) 16, which can be viewed as an extension of the Peikert-Waters PKE scheme into the proxy re-encryption setting. One key difference between the Peikert-Waters PKE construction and the PRE construction of [15] is that: all components in the ciphertext of the Peikert-Waters PKE are signed by the one-time signature (under the verification key $v k$ ), but the key component $c_{1}$ is not signed in the ciphertext of the PRE of [15]. Of course, signing $c_{1}$ can prevent our concrete attack, but the resultant scheme is not a PRE scheme any longer (particularly, the proxy cannot translate ciphertexts among players, as the underlying signing key w.r.t. $v k$ is unknown to the proxy). From our view, constructing CCA-secure proxy re-encryption without bilinear maps in the standard model may need significantly new ideas and techniques. It is still an open problem to come up with a (bidirectional or unidirectional) proxy reencryption scheme without bilinear maps in the standard model.

\section{Acknowledgements}

The first author is supported by the National Science Foundation of China under Grant Nos. 60903178 and 61005049, and it also supported by the Fundamental Research Funds for the Central Universities. The second author is supported in part by a grant from the Major State Basic Research Development (973) Program of China (No. 2007CB807901), and grants from the National Natural Science Foundation of China (Nos. 60703091 and 61070248) and the QiMingXing Program of Shanghai, and Young Faculty Fund of Computer Science School of Fudan University.

\section{References}

1. Ateniese, G., Fu, K., Green, M., Hohenberger, S.: Improved Proxy Re-Encryption Schemes with Applications to Secure Distributed Storage. In: NDSS. The Internet Society, San Diego (2005)

2. Ateniese, G., Fu, K., Green, M., Hohenberger, S.: Improved Proxy Re-Encryption Schemes with Applications to Secure Distributed Storage. ACM Trans. Inf. Syst. Secur. 9(1), 1-30 (2006)

3. Blaze, M., Bleumer, G., Strauss, M.: Divertible Protocols and Atomic Proxy Cryptography. In: Nyberg, K. (ed.) EUROCRYPT 1998. LNCS, vol. 1403, pp. 127-144. Springer, Heidelberg (1998)

4. Boyen, X., Waters, B.: Shrinking the keys of discrete-log-type lossy trapdoor functions. In: Zhou, J., Yung, M. (eds.) ACNS 2010. LNCS, vol. 6123, pp. 35-52. Springer, Heidelberg (2010)

5. Canetti, R., Goldreich, O., Halevi, S.: The Random Oracle Methodology, Revisited (Preliminary Version). In: STOC, pp. 209-218 (1998)

6. Canetti, R., Goldreich, O., Halevi, S.: The random oracle methodology, revisited. J. ACM 51(4), 557-594 (2004) 
7. Canetti, R., Hohenberger, S.: Chosen-Ciphertext Secure Proxy Re-Encryption. In: Ning, P., di Vimercati, S.D.C., Syverson, P.F. (eds.) ACM Conference on Computer and Communications Security, pp. 185-194. ACM, New York (2007)

8. Canetti, R., Krawczyk, H., Nielsen, J.B.: Relaxing Chosen-Ciphertext Security. In: Boneh, D. (ed.) CRYPTO 2003. LNCS, vol. 2729, pp. 565-582. Springer, Heidelberg (2003)

9. Chow, S.S.M., Weng, J., Yang, Y., Deng, R.H.: Efficient Unidirectional Proxy ReEncryption. In: Bernstein, D.J., Lange, T. (eds.) AFRICACRYPT 2010. LNCS, vol. 6055, pp. 316-332. Springer, Heidelberg (2010)

10. Deng, R.H., Weng, J., Liu, S., Chen, K.: Chosen-Ciphertext Secure Proxy Reencryption without Pairings. In: Franklin, M.K., Hui, L.C.K., Wong, D.S. (eds.) CANS 2008. LNCS, vol. 5339, pp. 1-17. Springer, Heidelberg (2008)

11. Freeman, D.M., Goldreich, O., Kiltz, E., Rosen, A., Segev, G.: More constructions of lossy and correlation-secure trapdoor functions. In: Nguyen, P.Q., Pointcheval, D. (eds.) PKC 2010. LNCS, vol. 6056, pp. 279-295. Springer, Heidelberg (2010)

12. Hemenway, B., Ostrovsky, R.: Lossy trapdoor functions from smooth homomorphic hash proof systems (2010) (manuscript), http://www.math.ucla.edu/ bretth/papers/uhp_ltdf.pdf

13. Libert, B., Vergnaud, D.: Unidirectional Chosen-Ciphertext Secure Proxy ReEncryption, http://hal.inria.fr/inria-00339530/en/ This is the extended version [14]

14. Libert, B., Vergnaud, D.: Unidirectional Chosen-Ciphertext Secure Proxy Reencryption. In: Cramer, R. (ed.) PKC 2008. LNCS, vol. 4939, pp. 360-379. Springer, Heidelberg (2008)

15. Matsuda, T., Nishimaki, R., Tanaka, K.: CCA Proxy Re-Encryption without Bilinear Maps in the Standard Model. In: Nguyen, P., Pointcheval, D. (eds.) PKC 2010. LNCS, vol. 6056, pp. 261-278. Springer, Heidelberg (2010)

16. Peikert, C., Waters, B.: Lossy trapdoor functions and their applications. In: Ladner, R.E., Dwork, C. (eds.) STOC, pp. 187-196. ACM, New York (2008)

17. Shao, J., Cao, Z.: CCA-Secure Proxy Re-encryption without Pairings. In: Jarecki, S., Tsudik, G. (eds.) PKC 2009. LNCS, vol. 5443, pp. 357-376. Springer, Heidelberg (2009)

18. Weng, J., Chen, M.-R., Yang, Y., Deng, R.H., Chen, K., Bao, F.: CCA-Secure Unidirectional Proxy Re-Encryption in the Adaptive Corruption Model without Random Oracles. Science China: Information Science 53(3), 593-606 (2010)

19. Weng, J., Deng, R.H., Liu, S., Chen, K.: Chosen-ciphertext secure bidirectional proxy re-encryption schemes without pairings. Inf. Sci. 180(24), 5077-5089 (2010) 